\title{
What's the Point? A Study of Full-Stop Use in Text Messages in Varying Emotional Contexts
}

\author{
Andrew Albritton ${ }^{1 *}$ \\ (iD) 0000-0003-3477-9078 \\ ${ }^{1}$ Missouri State University, USA \\ *Corresponding author: AndrewAlbritton@MissouriState.edu
}

Citation: Albritton, A. (2022). What's the Point? A Study of Full-Stop Use in Text Messages in Varying Emotional Contexts. Online Journal of Communication and Media Technologies, 12(1), e202203. https://doi.org/10.30935/ojcmt/11431

\section{ARTICLE INFO}

Received: 12 Aug 2021

Accepted: 4 Dec 2021

\begin{abstract}
Because of the pervasiveness of the text messaging medium, further understanding of meaningmaking practices in text messages is desirable. This study offers new knowledge of the potential uses and meanings of the full-stop in text messages. Although the full-stop has become infamous for its potential negative connotations in text messages, few studies have evaluated uses of the full-stop in varied emotional contexts. For this study, text message Discourse Completion Tasks (DCTs) detailing different emotional contexts were given to undergraduate students. The uses of full-stops in the text message responses elicited with the DCTs were evaluated via two methods of analysis. First, a basic content analysis was conducted for the purpose of understanding the relative uses of the full-stop in the differing emotional contexts. Second, a semiotic analysis was conducted to understand more fully how exactly the elicited fullstops were deployed and what meanings they might convey. It was concluded that the full-stop is not always a device with a negative connotation, and that, in certain circumstances, the fullstop may be seen as more appropriate than other punctuation marks. It may even be seen as a conveyer of formality, seriousness, or earnestness. The study offers practical knowledge of how a certain typographical device is used, as well as the more theoretical knowledge that punctuation devices, like words, can possess a certain semiotic elasticity: their uses and meanings can vary, depending on the contexts in which they are deployed and the people who write and read them.
\end{abstract}

Keywords: full-stops, punctuation marks, text messages, emotional expression

\section{INTRODUCTION}

Text messaging is an enormously popular medium of communication (Anderson, 2015; Heimlich, 2012). Given its popularity, further understanding of communicative practices within the medium is desirable. Texters use both verbal and nonverbal signs in constructing their messages (Albritton, 2017). Many recent studies have evaluated the use of emoji as a method of nonverbal meaning making in text messages (e.g., Bai et al., 2019; Danesi, 2016; Gawne \& McCulloch, 2019; Logi \& Zappavigna, 2021). There are, however, other interesting and important methods of nonverbal meaning making in text messages. This study seeks to elaborate understanding of the meaning making potential of the full-stop, which, while a rather humble mark in comparison with the colorfully and artistically rendered emoji, has nonetheless become a remarkable symbol. In the medium of text messaging, the full-stop has become more than a point of punctuation: it is now a point of contention because of its potential negative connotations. Popular articles on the full-stop's potential negativity have run in The New York Times (Bilefsky, 2016; Yagoda, 2012) and The New Republic (Crair, 2013). Scholars who have investigated full-stop use in text messages have found that full-stops are often omitted at the end of text messages because they are unnecessary (Baron, 2008; Baron \& Ling, 2011; Ling \& Baron, 2007) and that when they are included, they can convey negativity and a lack of sincerity (Gunraj et al., 2016; Houghton et al., 2018; Reynolds et al., 2017). In a medium famous for exuberant punctuation devices such as exclamation points and emoji, it is not hard to see how pragmatically unnecessary full-stops at the end of transmissions might come to be seen as unenthused, sarcastic, or even caustic. This study investigates

Copyright (c) 2022 by authors; licensee OJCMT. This article is an open access article distributed under the terms and conditions of the Creative Commons Attribution License (http://creativecommons.org/licenses/by/4.0/). 
other ways full-stops might function by observing how they are used in text messages in varying emotional contexts.

\section{METHODOLOGY}

Text message Discourse Completion Tasks (DCTs) (Albritton, 2020) were distributed to undergraduates at a Midwestern American university in the fall semester of 2013. The respondents were asked to send text message responses to five prompts:

1. You just heard that a good friend got a job he or she had been wanting. What would you text your friend? Please text your message to textresearch1@gmail.com.

2. You just heard that your friend did not get a job he or she had applied for. What would you text your friend? Please text your message to textresearch2@gmail.com.

3. You just heard that your friend got a new job, but you're not sure what he or she thinks about it. What would you text your friend? Please text your message to textresearch3@gmail.com.

4. You are part of a small group working on a project for a class. You think the group should have a meeting. What would you text the group's leader? Please text your message to textresearch4@gmail.com.

5. Please text your age and gender to textresearch5@gmail.com.

These prompts present four different contexts: the first is happy, the second is sad, the third is emotionally opaque, and the fourth is task-based. 41 students responded. For the purpose of easy citation of the dataset, each unique phone number was assigned a respondent number. 30 respondents gave their age and gender. Of those 30, the average age was 20.03 years and the median age was 20 years; 16 were male and 14 were female. 136 total text messages were collected. More information about the dataset can be found in Table 1.

It is worth briefly noting that very few of the text messages received ( $3.68 \%$ of them) contained no punctuation, a fact that indicates that punctuation can function as a normal and important part of meaning making in text messages.

The periods in the elicited text messages were analyzed according to two methods: First, a basic content analysis was conducted (Herring, 2004a, 2004b; Krippendorff, 1980) to get an overall understanding of relative uses of full-stops in each emotional context. Full-stops were distinguished as transmission medial or transmission final for this content analysis (Ling \& Baron, 2007). Second, the full-stops were analyzed semiotically that is, the punctuation marks were examined according to the speech acts they accompanied in order to determine what, if any, affect may be associated with them.

\section{CONTENT ANALYSIS}

The results of the content analysis are given in Table 2, along with tallies of the other punctuation marks received in response to the DCTs. The full-stop tallies are in bold type. Because each of the DCTs did not elicit the same number of text message responses from participants (see Table 1), averages of how many times a punctuation mark occurred per message are given in parentheses after the number of marks received in response to the DCT. Punctuation strings (e.g., !!! or ??, or even strings of emoji) were counted as single marks.

This content analysis suggests three points that are of importance. First, full-stops were deployed in responses to all of the DCTs. Because these DCTs would not call for sarcastic or negatively valence responses, we can conclude that the full-stop is not a device that is solely negative. Second, full-stops were used sparingly; and especially sparingly at the end of messages, further validating the conclusions of Baron (2008), Baron and Ling (2011), and Ling and Baron (2007). Third, more transmission medial and transmission final full-stops were used in response to the second DCT than the other DCTs. This second DCT posited that a friend of the respondent had not received a job that the friend had applied for, and would accordingly call for a consolatory response from the respondent. The greater number of transmission medial full-stops is probably due to the fact that other forms of punctuation may not have seemed as appropriate in breaking up the ideas within the responses because of the sad situation posited in the DCT. The greater number of transmission final full-stops in response to the second DCT is more interesting. Because these full-stops are not necessary to demonstrate 
Table 1. The dataset

\begin{tabular}{lcccc}
\hline & DCT 1 & DCT 2 & DCT 3 & DCT 4 \\
\hline Number of messages received & 37 & 34 & 34 & 31 \\
Average number of words in each message & 8.73 & 14.06 & 11.47 & 18.58 \\
Median number of words in each message & 9 & 11 & 11 & 17 \\
Messages without transmission-final punctuation & 8 & 6 & 3 & 8 \\
Messages without punctuation & 1 & 0 & 1 & 3 \\
\hline
\end{tabular}

the end of the transmission, their inclusion not only belies the idea of transmission final full-stops being always negative: it also suggests that the transmission final full-stop can, in such solemn and melancholy circumstances, convey an appropriate seriousness, formality, or even sincerity. This idea is further explored in the semiotic analysis below.

\section{SEMIOTIC ANALYSIS}

In the responses to the first DCT, three respondents used full-stops after congratulatory remarks: "Congrats on the job man. I'm going to come bother you at work ;)" (Respondent 2); "Congrats dude." (Respondent 30); and "Heard you got a new job. Congrats." (Respondent 32). While it is true that these congratulatory remarks may carry less buoyancy than the congratulatory remarks that are punctuated with exclamation marks, it would be wrong to argue that these messages are in some way inimical or sarcastic. The senders of these messages, rather, are sticking with more formal writing practices or are simply being less emotive, either by choice or by nature. Given the social context that was described in the DCT that elicited these messages, there is no reason to believe that these full-stops are mean-spirited in any way. The full-stop punctuated statements in which the senders affirmed that they had heard about the new job in three cases: "I heard you just got that job you wanted. Congratulations!" (Respondent 26); "Hey! Heard you got the job. Congrats! Glad everything worked out for you. :)" (Respondent 31); and "Heard you got a new job. Congrats." (Respondent 32); Respondent 31, in the cited message, also used a full-stop with a kind statement. Respondent 8 used the full-stop with a benediction: "Congrats dude! Good luck on yours first day." Respondent 34 placed a full-stop after a compliment (and offered a bit of advice): "Good work on getting the job man. Don't fuck it up". Respondent 9 used the full-stop with a greeting: "Hey dude. I heard you got the job! That's awesome!" The full-stops in these messages, like those above, may, in the minds of some readers, seem flat relative to constructions that include more excited punctuation; but, once again, such an interpretation is not a given, and it does not seem to be the case that these messages are in any way mean-spirited. (The profane counsel given in the latter message is clearly ludic and not unkind; in its own way, since it demonstrates a jocular and familiar tone, it would probably be viewed as friendly.)

Three respondents, it will be noticed, used the full-stop and the exclamation point in the same message. The messages produced by these respondents are of particular interest, because in them the exclamation mark and the full-stop are contrasted. The first comes from Respondent 8, who wrote, "Congrats dude! Good luck on your first day.". This respondent shows enthusiasm in his felicitations, and a bit more restraint in the offer of good fortune that follows. Perhaps this restraint shows some seriousness and earnestness in regards to the wish for good luck - or perhaps it is simply a way to standardly end a sentence that is not meant as an exclamation. In any case, there is a contrast here between the first and second sentences of the message and the contrast could be viewed as mimicking vocal expression. The second example comes from Respondent 9, who wrote, "Hey dude. I heard you got the job! That's awesome!". This respondent's greeting has a full-stop, while the following statements about the new job are exclaimed. Thus Respondent 9 offers a less excited greeting, but evinces excitement in regards to the new job the message's receiver has acquired. The third comes from Respondent 31, who wrote, "Hey! Heard you got the job. Congrats! Glad everything worked out for you. :)". In this message, the greeting and congratulatory remarks are rendered as exclamations; and the statement of fact about hearing about the new job, and the remark concerning the sender's happiness for the receiver, are punctuated with the soberer full-stop; the latter remark (the one about being happy for the recipient of the message), may be an example of a standard usage of the full-stop, or it may, through its formality, show some seriousness on the part of the sender. These three messages hint at the potential vocal element of the full-stop: It can demonstrate to the mind of readers a less exclamatory 
Table 2. Content analysis result

\begin{tabular}{lcccc}
\hline & DCT 1 & DCT 2 & DCT 3 & DCT 4 \\
\hline Exclamation points & $37(1.00)$ & $14(0.41)$ & $9(0.26)$ & $6(0.19)$ \\
Question marks & $5(0.14)$ & $5(0.15)$ & $25(0.74)$ & $18(0.58)$ \\
Transmission-medial full-stops & $\mathbf{7 ( 0 . 1 9 )}$ & $\mathbf{2 2 ( 0 . 6 5 )}$ & $\mathbf{1 0 ( 0 . 2 9 )}$ & $\mathbf{1 2 ( 0 . 3 9 )}$ \\
Transmission-final full-stops & $\mathbf{3 ( 0 . 0 8 )}$ & $\mathbf{1 1 ( 0 . 3 2 )}$ & $\mathbf{1 ( 0 . 0 3 )}$ & $\mathbf{4 ( 0 . 1 3 )}$ \\
Total full-stops & $\mathbf{1 0 ( 0 . 2 7 )}$ & $\mathbf{3 3 ( 0 . 9 7 )}$ & $\mathbf{1 1 ( 0 . 3 2 )}$ & $\mathbf{1 6}(\mathbf{0 . 5 2 )}$ \\
Emoticons & $5(0.14)$ & $6(0.18)$ & $2(0.06)$ & $0(0.00)$ \\
Emoji & $1(0.03)$ & $0(0.00)$ & $0(0.00)$ & $0(0.00)$ \\
Strings & $12(0.32)$ & $4(0.12)$ & $9(0.26)$ & $2(0.06)$ \\
Ellipses & $0(0.00)$ & $1(0.03)$ & $1(0.03)$ & $0(0.00)$ \\
Commas & $5(0.14)$ & $21(0.62)$ & $10(0.29)$ & $12(0.39)$ \\
Quotation marks & $0(0.00)$ & $0(0.00)$ & $1(0.03)$ & $0(0.00)$ \\
Asterisks & $1(0.03)$ & $0(0.00)$ & $1(0.03)$ & $0(0.00)$ \\
Slash & $0(0.00)$ & $0(0.00)$ & $0(0.00)$ & $1(0.03)$ \\
XO & $0(0.00)$ & $1(0.03)$ & $0(0.00)$ & $0(0.00)$ \\
\hline
\end{tabular}

tone, especially when it stands in a text with an exclamation mark. The fact that it can carry this vocalic meaning has no doubt contributed to the feeling that it can seem to be flat or harsh. However, it is worth remembering that serious and earnest vocal tones are often flatter than exclamatory ones, and the last cited full-stop above could very well be a way of showing earnestness of sentiment, as suggested in the content analysis section. Even if, in the mind of the sender, the contrasted exclamation point and full-stop are not meant to signify disparate levels of vocal excitement, these marks at the very least do evince differing levels of emphasis or cognitive excitement in regards to the things being written in the message. In other words, even if these marks are not expressly meant to stand in for vocal performance, they do stand for what vocal performance would demonstrate if the messages were spoken aloud - namely, differing levels of emphasis and excitement on the part of the sender. In any case, notwithstanding this disparity in meaning, it does not appear here that the cited full-stops have a negative meaning.

In the responses to the second DCT, the full-stop was used relatively frequently. It was used twenty-eight times to punctuate different kinds of consoling and encouraging statements and imperatives. To give some examples: "I'm sorry buddy, hang in there. There's better jobs any way." (Respondent 3); “Dang, that sucks. I'm sorry. Keep your head up, and keep looking." (Respondent 15); "I'm sorry friend. Ik GOD has sum better instore for you." (Respondent 11); and "Awe :( I'm sorry. They suck anyway." (Respondent 33). Four respondents punctuated statements about hearing the news with a full-stop; for example, "Hey girl, I heard that you didn't get the job. I'm sorry. :( Lets get together soon though and forget about it." (Respondent 40). Respondent 9 punctuated a greeting with a full-stop: "Hey man. Sorry about the job. It'll work out." The medial full-stops in these examples are performing their normal structural function of creating a high level of disjunction between ideas. The fact that they are used more frequently here is not surprising, since, in the context created by the second DCT, a more solemn tone, and fewer questions, would be expected: People wanted to send multiple thoughts in their messages, and they used the standard method of separating those thoughts: the full-stop.

The transmission-final full-stops that are used in these and the other messages that were sent in response to DCT 2 are certainly not caustic or terse. As asserted in the content analysis, their frequency in these somber messages - when they could easily have been omitted - demonstrates that the senders are including them for some reason. Perhaps they are simply included out of habit. But, as asserted above, it is possible that the standard formal nature of the mark can signify seriousness and earnestness. In any case, they show that there is a place for the full-stop in text messages: in more serious and consolatory circumstances. There were, as seen in Figure 2, also a substantial number of exclamation points sent in response to the second DCT, suggesting that the exclamation point can be used to emphasize positive sentiments and show enthusiasm for positive ideas, even in the midst of somber circumstances. The full-stop, on the other hand, may show less enthusiasm, but its relative flatness of tone is seen by some as suitable in conjunction with certain ideas and speech acts.

In some messages, one or more full-stops were used as well as one or more exclamation points; these messages with both punctuation marks in them, like those discussed previously, show some distinction between the meanings of the two marks, so they are worthy of some special attention. Respondent 13 wrote, 
"Hey Alexis, I heard about the job mishap. Its their loss though haha. You'll definitely get the next one!!" In this message, after the "job mishap" is mentioned in the statement of the opening sentence, the full-stop is used. It creates the normal disjunction between the first and second sections of text, and, following this remark concerning an inauspicious turn of events, an exclamation point would be out of place - the full-stop, however, is appropriate. In the second sentence, the sender introduces some levity, writing a friendly sentiment followed by a simulated laugh and concluding with a full-stop. In this sentence, an exclamation mark after the "haha" might indicate a more boisterous laugh, which, in this situation, would be uncalled for. The full-stop could be viewed as mitigating the force of the laugh, which was meant in the first place just to show some good feeling toward the recipient of the message, and not outright hilarity. The final sentence of the message presents a positive prognostication regarding the recipient's future opportunities, followed by a string of two exclamation marks, thereby showing enthusiasm for the potential of the recipient and the recipient's forthcoming professional prospects. Respondent 16 wrote, “Hey sweetheart, heard you didn't get the job. But here's the thing, everything happens for a reason! I know you probably don't see it that way right now, but this may lead you to different opportunities that you may have not seen before. Keep your head up! I mean it when I say that they are missing out on a fabulous employee". Here, the exclamation point is used to give emphasis, or force, to certain thoughts, while the full-stop is used as a punctuation device to separate ideas that do not require the same force. The notification that the sender heard the bad news about the job doesn't need to be emphasized in any way, but the ancient aphorism "everything happens for a reason" is given emphasis, since it could help the person to put things into a broader perspective and feel better. The sentence following the aphorism is an explanatory statement, in that it expounds upon how the aphorism applies to the situation in question, and it is concluded with a full-stop, a natural punctuation mark for a more contentrich sentence. Then there is the imperative appeal to maintain a positive attitude ("Keep your head up!"); the exclamation mark following this sentence gives added emphasis to the sender's exhortation. The final sentence is not punctuated; perhaps the mark was excluded because of its superfluity, or perhaps it was left out because of its reputation of harshness, or perhaps it was forgotten. This rather extended message shows us once more that the exclamation point can be used to give emphasis to ideas, and the full-stop, when used in the same message, can give less force, or less emphasis. Respondent 28 , it should be noted, sent a similar message: "Hey girl I'm sorry about the job, just remember everything happens for a reason. You'll get the next one!". This respondent chose to punctuate the aphorism with a full-stop, and then gave the encouraging remark an exclamation point; this message, when compared with the one sent by Respondent 16, serves as a vital reminder that people will not always use the same punctuation marks with the same types of phrases - they will not always choose to emphasize the same types of thoughts or express the same levels of emotion, even if they do compose similar messages.

In the responses to DCT 3, six full-stops were used after sentences in which respondents claimed to have heard about the recipient's new job; for example, "Hey! I heard you got a new job. How do you like it?" (Respondent 9); "Hey Sarah, I heard you got a new job. I hope the first day goes well!!" (Respondent 13); and "Hey, i just heard that you got a new job. How do you like it?" (Respondent 35). These full-stops seem to serve simply as a means of separating ideas within the messages. In two cases, the full-stop was used after congratulatory remarks: "Congrats on it new job. How r u feelin about it??" (Respondent 11) (this message was followed by a corrective reading "* $U r$ " that is meant to replace the "it" in the original message); and "Hey heard about the new job. Congrats. You excited?" (Respondent 36). These full-stops separate ideas, and they, like those sent in the purely congratulatory messages elicited by DCT 1, might be read by some as somewhat flat - but, again, most people would probably be gratified to receive the congratulatory remark, no matter the punctuation. Furthermore, these felicitations may be punctuated with the flatter mark because of the affective opacity of the situation given in the DCT. One person used full-stops in a message that used statements to inquire indirectly about the new job and express a hope that things were going well: "Hey, just wondering how the new job is going. Hopefully everything is falling into place." (Respondent 31). Another respondent - who it would seem misunderstood the DCT and read it as meaning that the new job was not really being enjoyed by the hypothetical friend in question - used the full-stop to punctuate an imperative sentence encouraging the receiver of the message to keep working at the job to see if things would improve: "Just stay in it and see how everything goes for awhile and if its not getting better than quit. But just try to keep an open mind" (Respondent 27). 
In the messages elicited by DCT 4, the full-stop was used twelve times in conjunction with statements of the sender's belief that a meeting should be convened. Examples include: "Hello, we need to get the group together for a meeting this week. Lets set up a time and place?" (Respondent 8); "Hey! I really think we ALL need to get together for this project soon. Text or call me about a time that works and we'll round up the group." (Respondent 9); "Hey group leader, I think we need to get together and discuss some things about the project." (Respondent 10); "Hey I think we should have a meeting soon.” (Respondent 11); “Hey, so I know we really need to start getting stuff done on this research project and I think we should try setting up a time/place for a group meeting. Can you text everyone else and see whatll work best for them?" (Respondent 13); “Hey I was thinking that we should probably meet up as a group. If you need help figuring out when and where to meet, let me know and ill help you" (Respondent 16); "Hey we need to get together for this project. Does right after class work for everyone?" (Respondent 33); "Hey, i think we should meet up sometime so we can work on the project." (Respondent 35); and "Hey how's it goin? I think we need to get the group together for a meeting to go over some stuff with the project. What do you think?" (Respondent 36). Respondent 9 used a full-stop with a different type of statement: "Hey! I really think we ALL need to get together for this project soon. Text or call me about a time that works and we'll round up the group." This message's final full-stop punctuates a sentence that has two independent clauses, the first of which is an imperative and the second of which is a statement. The statement, the speech act directly punctuated by the full-stop, is about a future action predicated upon the completion of the imperative of the sentence's first independent clause. And the future action, moreover, is an action the message-recipient will take (along with the message-sender). Thus the ultimate thrust of the sentence is imperative. One writer used a full-stop with a greeting: "Hey you guys. I believe that we need to have a meeting about this project" (Respondent 34). Two respondents coupled fullstops with statements that claimed their identity; for example, "Hey it's [student's name] from the cis class. Do you think our group should try to meet up sometime to talk about the project??" (Respondent 38). The punctuation style in the responses to this fourth DCT were more standard and less emotive, which is to say, there were relatively fewer exclamation points and punctuation strings in the messages, and there were no emoticons or emoji. In the above examples, we see that the respondents frequently stated what they were thinking, and they used full-stops to punctuate these ideas. The DCT called for a task to be accomplished - a task lacking any heavy emotional weight - and people sent text-messages that were less emotive and more task-based, and thus they used these full-stops. All of this is not, of course, surprising - but it is worthy of remark, because it shows that the medium of text messaging is not one of incessant pictographs, exclamations, and non-standard symbols and constructions. In certain situations, some people will change their style of composition to make it more standard, and more standard constructions will involve more fullstops.

\section{CONCLUSIONS}

Semiotic interpretation in a study such as this one must be done with a care, and so it should be plainly stated that literally every single message-medial usage of full-stops in this study's data could be viewed simply as separating ideas standardly and in a non-exclamatory and non-interrogative way, just as every messagefinal full-stop could be viewed as ending the message standardly and in a non-exclamatory and noninterrogative way. There is not, in other words, any necessary or abundantly obvious emotional meaning attached to the full-stop that can be inferred. However, there are some important conclusions that can be drawn based on both the content analysis and the semiotic analysis. The full-stop does contrast with the exclamation point in a fairly clear manner, in that it shows less excitement and less emphasis. It may also be concluded that the full-stop is not a mark that is singularly hostile or insincere; while it is possible that it can be used to convey enmity, this study's data shows that it is used in messages to friends that are intended to be congratulatory and consoling. It is possible that the formality of the full-stop, along with its relative emotional flatness, may be employed by certain writers as a way to show seriousness, thoughtfulness, or earnestness. In recognizing these disparate ways in which the full-stop might be read, we are reminded that the art of interpretation, even in respect to punctuation marks, can be tricky. Across contexts, some people will use and read certain punctuation marks one way, and some people will use and read them another. But even this point is important, because it illumines the fact that punctuation marks are not entities of discrete and concrete meaning - they vary in their connotations from context to context and from person to person. 
They possess core meanings, but these meanings can bend, depending upon the situation and the interlocutors. A full-stop may in fact be used as a means of communicating terseness or an end to a conversation, or it might be used as a means of ending a message standardly, formally, or earnestly; and it can be read in either way, so writers must be aware of the potential meanings they are constructing, and must endeavor to ensure that the meaning they wish to send is the one that is most evident in a message. One way to accomplish this aim would be to use lucid language that makes the intent of the sender clear - the sender could, for instance, simply articulate their emotional state, and then the punctuation mark would not bear the entire burden of carrying the emotional meaning (see Albritton, 2017).

Funding: Author received no financial support for the research and/or authorship of this article.

Declaration of interest: Author declares no competing interest.

Data availability: Data generated or analysed during this study are available from the author on request.

\section{REFERENCES}

Albritton, A. (2017). Emotions in the ether: Strategies for effective emotional expression in text-messages. Online Journal of Communication and Media Technologies, 7(2), 50-58. https://doi.org/10.29333/ojcmt/2590

Albritton, A. (2020). The text-message discourse completion task: A method for the collection of textmessages. International Journal of Language and Linguistics, 7(1), 125-133. https://doi.org/10.30845/ijll.v7n1p14

Anderson, M. (2015). For teens, phone calls are reserved for closer relationships. Pew Research Center. https://www.pewresearch.org/fact-tank/2015/08/17/for-teens-phone-calls-are-reserved-for-closerrelationships/

Bai, Q., Dan, Q., Mu, Z., \& Yang, M. (2019). A systematic review of emoji: Current research and future perspectives. Frontiers in Psychology, 10, 2221. https://doi.org/10.3389/fpsyg.2019.02221

Baron, N. S. (2008). Always on: Language in an online and mobile world. Language Learning \& Technology, 14(3), 19-22. https://doi.org/10.1093/acprof:oso/9780195313055.001.0001

Baron, N., \& Ling, R. (2011). Necessary smileys \& useless periods. Visible Language, 45(1/2), 45-67. http://visiblelanguage.herokuapp.com/issue/152/article/843

Bilefsky, D. (2016, June 9). Period. Full stop. Point. Whatever it's called, it's going out of style. The New York Times. https://www.nytimes.com/2016/06/10/world/europe/period-full-stop-point-whatever-its-calledmillennials-arent-using-it.html

Crair, B. (2013, November 25). The period is pissed: When did our plainest punctuation mark become so aggressive? New Republic. http://www.newrepublic.com/article/115726/period-our-simplestpunctuation-mark-has-become-sign-anger

Danesi, M. (2016). The semiotics of emoji: The rise of visual language in the age of the Internet. Media and Communication, 5(4), 75. https://doi.org/10.17645/mac.v5i4.1041

Gawne, L., \& McCulloch, G. (2019). Emoji as digital gestures. Language@Internet, 17, article 2. https://www.languageatinternet.org/articles/2019/gawne/index_html

Gunraj, D. N., Drumm-Hewitt, A. M., Dashow, E. M., Upadhyay, S. S. N., \& Klin, C. M. (2016). Texting insincerely: The role of the period in text messaging. Computers in Human Behavior, 55, 1067-1075. https://doi.org/10.1016/j.chb.2015.11.003

Heimlich, R. (2012). Texting is nearly universal among young adult cell phone owners. Pew Research Center. https://www.pewresearch.org/fact-tank/2012/12/14/texting-is-nearly-universal-among-young-adultcell-phone-owners/

Herring, S. C. (2004a). Computer-mediated discourse analysis: An approach to researching online communities. In S. A. Barab, R. Kling, \& J. H. Gray (Eds.), Designing for virtual communities in the service of learning (pp. 338-376). Cambridge University Press. https://doi.org/10.1017/CBO9780511805080.016

Herring, S. C. (2004b). Content analysis for new media: Rethinking the paradigm. In New research for new media: Innovative research methodologies symposium working papers and readings (pp. 47-66). University of Minnesota School of Journalism and Mass Communication. http://ella.slis.indiana.edu/ herring/newmedia.pdf 
Houghton, K. J., Upadhyay, S. S. N., \& Klin, C. M. (2018). Punctuation in text messages may convey abruptness. Period. Computers in Human Behavior, 80, 112-121. https://doi.org/10.1016/j.chb.2017.10.044

Krippendorff, K. (1980). Content analysis: An introduction to its methodology. SAGE.

Ling, R., \& Baron, N. S. (2007). Text messaging and IM: Linguistic comparison of American college data. Journal of Language \& Social Psychology, 26(3), 291-298. https://doi.org/10.1177/0261927X06303480

Logi, L., \& Zappavigna, M. (2021). A social semiotic perspective on emoji: How emoji and language interact to make meaning in digital messages. New Media \& Society. https://doi.org/10.1177/14614448211032965

Reynolds, K., Casarotto, B., Noviski, S., \& Roche, J. (2017). Using punctuation as a marker of sincerity and affective convergence during texting. In CogSci 2017 Proceedings (pp. 2986-2991). https://cogsci.mindmodeling.org/2017/papers/0565/paper0565.pdf

Yagoda, B. (2012, August 6). The point of exclamation. The New York Times: The Opinion Pages. http://opinionator.blogs.nytimes.com/2012/08/ 06/the-point-of-exclamation/

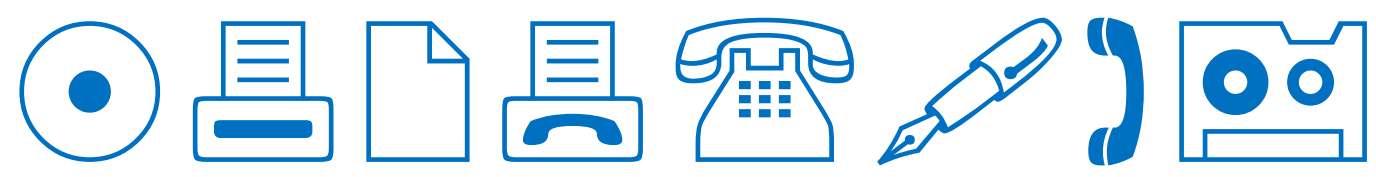

